\title{
Rational In Silico Design of an Organic Semiconductor with Improved Electron Mobility
}

\author{
Pascal Friederich, Verónica Gómez, Christian Sprau, Velimir Meded, Timo Strunk, \\ Michael Jenne, Andrea Magri, Franz Symalla, Alexander Colsmann, \\ Mario Ruben, * and Wolfgang Wenzel*
}

Organic semiconductors find a wide range of applications, such as in organic light emitting diodes, organic solar cells, and organic field effect transistors. One of their most striking disadvantages in comparison to crystalline inorganic semiconductors is their low charge-carrier mobility, which manifests itself in major device constraints such as limited photoactive layer thicknesses. Trial-and-error attempts to increase charge-carrier mobility are impeded by the complex interplay of the molecular and electronic structure of the material with its morphology. Here, the viability of a multiscale simulation approach to rationally design materials with improved electron mobility is demonstrated. Starting from one of the most widely used electron conducting materials $\left(\mathrm{Alq}_{3}\right)$, novel organic semiconductors with tailored electronic properties are designed for which an improvement of the electron mobility by three orders of magnitude is predicted and experimentally confirmed.

as its synthetic accessibility. Yet, its comparably low electron mobility of $1.1 \times 10^{-10}$ $3.7 \times 10^{-7} \mathrm{~cm}^{2} \mathrm{~V}^{-1} \mathrm{~s}^{-1},{ }^{[3]}$ which is at least three orders of magnitude lower than the hole mobility of typical hole transporting materials such as $\alpha$-NPD, ${ }^{[3]}$ have motivated intense but costly and protracted experimental efforts to develop better ETL materials. ${ }^{[4,5]}$ So far, however, these materials often show lower electron affinities compared to $\mathrm{Al}_{3},{ }^{[4]}$ which leads to unbalanced charge carrier injection and hence reduced device efficiencies. Development of better materials, thus, requires systematic improvement of their intrinsic charge carrier mobility under various additional constraints, such as energy level compatibility with optimized OLED architectures,

Improvement of the charge carrier mobility of functional materials in organic light emitting diodes ${ }^{[1]}$ (OLEDs) is directly connected to their efficiency, as higher mobility leads to lower resistivity, improved better charge balance, and lower power consumption for a given applied electric field. Although today a wide range of hole transport materials has become available, Tris-(8-hydroxyquinoline)aluminum $\left(\mathrm{Alq}_{3}\right)^{[2]}$ has been used as a reference material for electron transport layers (ETLs) in OLEDs because of its high electron affinity, its stability as well

Dr. P. Friederich, Dr. V. Gómez, ${ }^{[+]}$Dr. V. Meded, Dr. T. Strunk,

Dr. A. Magri, ${ }^{[+]}$Dr. F. Symalla, Prof. M. Ruben, Prof. W. Wenzel

Karlsruhe Institute of Technology

Hermann-von-Helmholtz-Platz 1

76344 Eggenstein-Leopoldshafen, Germany

E-mail: mario.ruben@kit.edu; wolfgang.wenzel@kit.edu

C. Sprau, M. Jenne, Dr. A. Colsmann

Karlsruhe Institute of Technology

Kaiserstraße 12, 76131 Karlsruhe

Prof. M. Ruben

CNRS-Université de Strasbourg

23, rue du Loess, BP 43, 67034 Strasbourg cedex 2, France

The ORCID identification number(s) for the author(s) of this article can be found under https://doi.org/10.1002/adma.201703505.

[+]Present address: Palacký University Olomouc, Kř́žkovského 8, 77147 Olomouc, Czech Republic

${ }^{[++]}$Present address: MIXER Spa, Via Chiara 6/C, 48012 Villa Prati di Bagnacavallo, Italy processing, and charge balancing requirements. Presently, some of the most prominent solutions to solve these problems comprise a spectrum of doping techniques, ${ }^{[6]}$ which entails the use of materials mixtures in the device. In order to tackle this challenge, many materials aspects must be addressed in a scalebridging approach that incorporates transport models in amorphous organic semiconductors (see Bässler and co-workers, ${ }^{[7,8]}$ extensions of these models, ${ }^{[9]} \mathrm{KMC}$ techniques, ${ }^{[10]}$ and multiscale modeling approaches ${ }^{[11,12]}$ ).

In this work, we use a multiscale modeling approach to calculate the electron mobility of new aluminum complexes derived from the well-known $\mathrm{Alq}_{3}$. Systematic analysis of the compounds as shown in Figure 1a-d enable us to deduce design rules for enhanced electron mobility under the constraint of energy level preservation. We apply these rules to develop the materials shown in Figure 1e with predicted electron mobility outperforming the electron mobility of $\mathrm{Alq}_{3}$ by up to three orders of magnitude. We identify and synthesize the most promising candidate $\mathrm{Al}_{2} \mathrm{MHept}$ and confirm the theoretical findings using space charge limited current (SCLC) experiments.

In the multiscale simulation protocol, we first generate a morphology model of the material based on molecule-specific force fields, ${ }^{[13]}$ with molecular geometries and partial charges parameterized by B3-LYP/def2-SV(P) density functional theory (DFT) calculations. ${ }^{[14]}$ Internal dihedral potentials are parameterized using the semiempirical PM6 functional as implemented in MOPAC. ${ }^{[15]}$ We use a molecular simulation approach mimicking vapor deposition ${ }^{[13]}$ to generate atomistic thin film 
a)

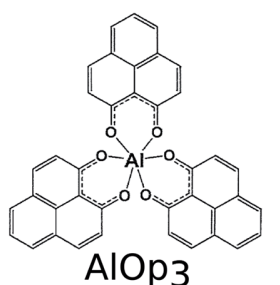

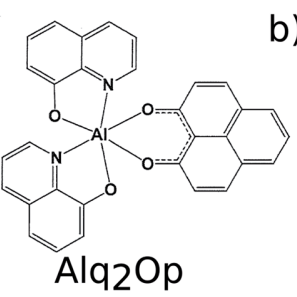

b)

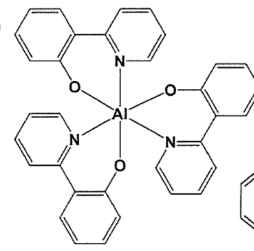

AlPopy3

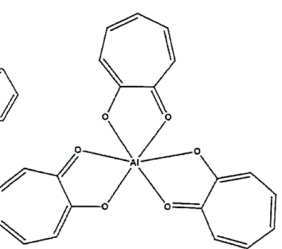

AlTrop3

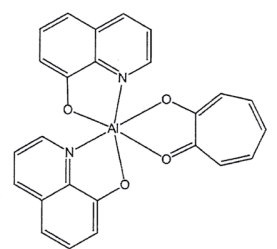

Alq2Trop

C)

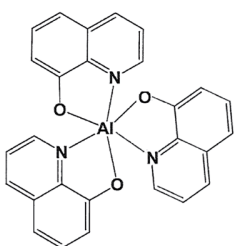<smiles>CC1CC(C)OC2(OC3=CC=CC4=C2N3N=CC=C4)O1</smiles><smiles></smiles><smiles></smiles><smiles>[GeH3]</smiles><smiles></smiles>

AlqAcac2

AlqAcac3

d)

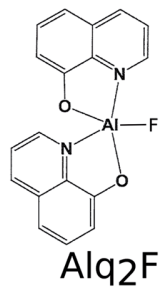

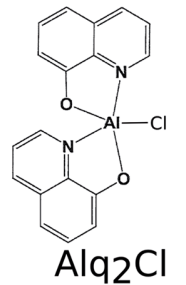

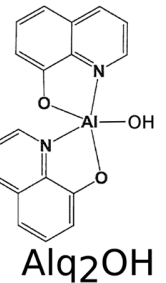

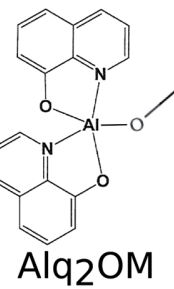

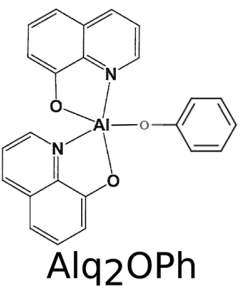

e)
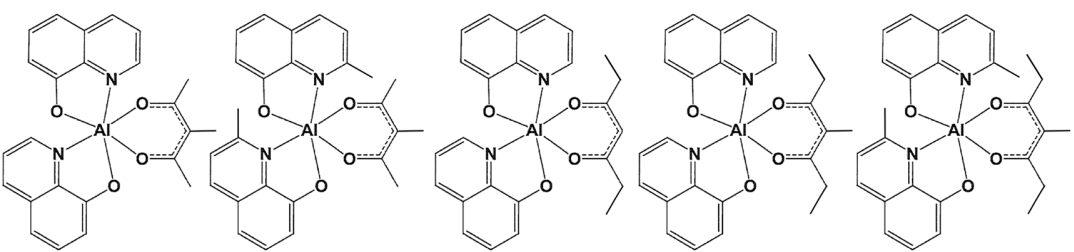

Alq2MAcac Alqm2MAcac Alq2Hept Alq2MHept Alqm2MHept

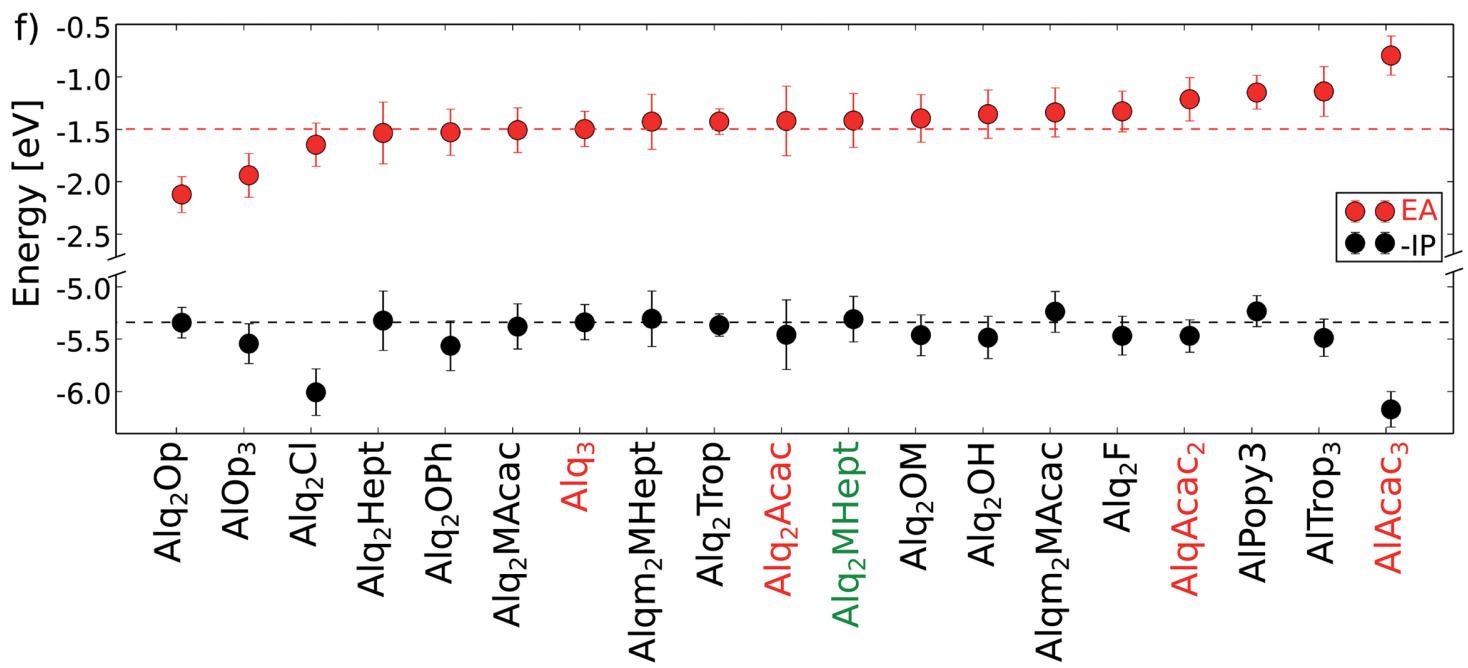

Figure 1. a-e) The aluminum complexes studied in this work are presented. a,b) Molecules where at least one of the three quinoline ligands of Alq ${ }_{3}$ was interchanged by ligands with larger or smaller conjugated $\pi$-systems, respectively. c) The systematic exchange of quinoline ligands of Alq $q_{3}$ by Acac ligands. d) $A q_{2} X$ molecules where the interchanged ligand $X$ was chosen to minimize the dipole moment and e) $A l q_{2} X$ molecules where $X$ was chosen to minimize the electrostatic potential created by the molecule. f) The calculated bulk IP and EA energies for all tested materials. The dashed horizontal lines indicate the IP/EA gap of the reference material $\mathrm{Alq}_{3}$. The materials $\mathrm{Alq}_{3}$ to $\mathrm{AlAcac}_{3}(\mathrm{see}(\mathrm{c})$ ) are marked in red as an illustration of the systematic increase of the EA (see text). Alq $q_{2} \mathrm{MHept}$ with the largest simulated electron mobility is marked in green. Bulk HOMO and LUMO energies can be found in Figure S2 (Supporting Information). 
morphologies containing 1000 molecules per system, which are periodically extended in $x$ - and $y$-direction. A detailed analysis of the atomistic systems can be found in Figure S1 of the Supporting Information. Based on these morphologies, we compute distributions of the polaron levels based on bulk ionization potentials (IP) and electron affinities (EA) using the Quantum Patch method, ${ }^{[16,17]}$ which accounts for polarization effects due to additional electrons and holes self-consistently at the DFT level. Electronic couplings are calculated using the Löwdin orthogonalization ${ }^{[18]}$ in electrostatically self-consistent, neutral systems. From the latter, the dipole moments of molecules embedded in the morphology are extracted. Reorganization energies are estimated using Nelsens four-point-procedure. ${ }^{[19]}$ The zero-field electron mobility is then computed for each material in the generalized effective medium model approximation. ${ }^{[20]}$

In addition to the mobility, the on-site energy of charge carriers is a key parameter determining charge carrier injection and driving voltage of organic semiconductor devices. For ETL, the electron affinity has to be closely aligned with the work function of the electron injecting electrode as any significant energetic misalignment would lead to either large energy barriers or substantial energy losses. Calculated IPs/EAs as well as highest occupied/lowest unoccupied molecular orbital (HOMO/LUMO) energies of amorphous thin-films are shown in Figure 1f. Polarization effects due to the local and global environment modify IP and EA levels and, to a smaller extent, even HOMO/LUMO energies. In order to accurately determine these levels and their distribution, a detailed quantum-mechanical treatment of the polarization in the bulk is required.

We observe a relation between the size of the largest conjugated $\pi$-system of a molecule and its energy levels (see Figure $1 \mathrm{f}$ and Figure S2 as well as the discussion in the Supporting Information). All materials having at least one quinoline-ligand as largest conjugated electronic system show comparable IPs and LUMO levels as the reference molecule, whose IP/EA gap is indicated as dashed lines. Observed stronger delocalization of molecular orbitals on, e.g., the Op ligand compared to quinoline leads to larger electron affinities and lower LUMO energies. The opposite effect can be observed in the very small conjugated system of, e.g., the Acac ligand, leading to the low electron affinity of $\mathrm{AlAcac}_{3}$ and a shallow LUMO level. This behavior is systematically analyzed upon the stepwise interchange of quinoline by $\mathrm{Acac}\left(\mathrm{Alq}_{3}, \mathrm{Alq}_{2} \mathrm{Acac}, \mathrm{AlqACac}_{2}\right.$, and $\mathrm{AlAcac}_{3}$, indicated in red in Figure 1f). Upon interchange of a single quinoline ligand, the EA shift is smaller than $0.2 \mathrm{eV}$, whereas the step from $\mathrm{AlqAcac}_{2}$ to $\mathrm{AlAcac}_{3}$ decreases the electron affinity by more than $0.5 \mathrm{eV}$. As a first design rule for new organic semiconductors, we want to keep IP and EA levels constant by exploiting charge localization on the largest $\pi$-conjugated molecular subsystems. IP and EA preserving single ligand substitution can, thus, be used to improve the charge carrier mobility by specific design of the interchanged ligand.

The performance of a device intrinsically depends on the charge carrier mobility, which exponentially depends on the square of the energy disorder of the constituting materials. ${ }^{[10,20]}$ It is generally accepted that apart from internal distortions of the molecules in the amorphous phase, one of the main sources of energy disorder are the molecular dipole moments. ${ }^{[7,12]}$ As Figure 2a demonstrates, consideration of the dipole moment alone is insufficient to fully rationalize the mobility as a function of composition. Several outliers (marked in red and illustrated in Figure 1d) show a high energy disorder while having a comparably low molecular dipole moment. While the interchange of a single ligand reduces the overall molecular dipole moment in these materials, the ligand fails to screen the polar core of the molecule causing substantial intermolecular electrostatic interaction, which results in high energy disorder. This is illustrated by the analysis of the mean electrostatic potential in close to medium proximity to the molecule. As shown in Figure 2b, energy disorder $\sigma$ and electron mobility $\mu$ are both strongly correlated with the mean interaction potential. The strongest correlation with a correlation coefficient of $r=0.80$ is achieved by averaging the electrostatic potential of the molecule on its accessible surface areas with distances between 3.2 and $4.6 \AA$ to the atom positions. We note that the mean electrostatic potential can be fine-tuned by adjusting the interchanged ligand, while keeping the electron affinity essentially constant. A decrease of the mean electrostatic potential diminishes the energy disorder of a given material and thus boosts the corresponding charge carrier mobility orders of magnitude. This can be formulated into a second design rule: high charge mobility requires molecules with a low mean electrostatic potential.

Using the second design rule, we systematically varied the third ligand of $\mathrm{Alq}_{3}$ to decrease the mean interaction potential from $140 \mathrm{mV}$ for $\mathrm{Alq}_{3}$ to $67 \mathrm{mV}$ for Alqm${ }_{2}$ MHept (Figure 2b). The resulting compounds are displayed in Figure 1e. We have computed the electron mobility of all of these molecules using the same approach and find $\mu=1.4 \times 10^{-4} \mathrm{~cm}^{2} \mathrm{~V}^{-1} \mathrm{~s}^{-1}$ for the most promising candidate $\left(\mathrm{Al}_{2} \mathrm{MHept}\right)$ (see Figure $2 \mathrm{c}$ ). The additional methyl group at the central carbon atom of the interchanged ligand decreases the dipole moment while the elongation of the alkyl chain from five to seven carbon atoms improves the shielding of the molecular core. The low electron affinity of $\mathrm{AlAcac}_{3}$ disobeys the first design rule, which is why we discard it as an improved ETL candidate.

We have confirmed the theoretical prediction by synthesis of $\mathrm{Alq}_{2} \mathrm{MHept}$ followed by preparation of spin-coated devices (see Figure 3a and Figure S3 in the Supporting Information) and SCLC measurements (see Figures $2 \mathrm{c}$ and $3 \mathrm{~b}$ and Figure S4 in the Supporting Information). The electron mobility $\mu$ is derived from the quadratic regime $\left(I \sim U^{2}\right)$ of the SCLC measurements using Equation (1)

$$
I=\frac{9}{8} A \varepsilon_{0} \varepsilon \mu \frac{U^{2}}{d^{3}}
$$

where $I$ is the measured current, $A=0.035 \mathrm{~cm}^{2}$ is the device area, $\varepsilon_{0}$ is the vacuum permittivity, $\varepsilon \approx 3$ is the relative permittivity, ${ }^{[21]} U$ is the applied voltage, and $d$ is the layer thickness obtained from surface profilometer measurements $\left(d=150 \mathrm{~nm}\right.$ for $\mathrm{Alq}_{3}, d=185 \mathrm{~nm}$ for $\mathrm{Alq}_{2} \mathrm{Acac}$, and $d=250 \mathrm{~nm}$ for $\mathrm{Alq}_{2} \mathrm{MHept}$ ). Equation (1) yields an electron mobility of $\mu=6.5 \times 10^{-8} \mathrm{~cm}^{2} \mathrm{~V}^{-1} \mathrm{~s}^{-1}$ for $\mathrm{Alq}_{3}, \mu=1.1 \times 10^{-5} \mathrm{~cm}^{2} \mathrm{~V}^{-1} \mathrm{~s}^{-1}$ for $\mathrm{Alq}_{2} \mathrm{Acac}$, and $\mu=3.3 \times 10^{-4} \mathrm{~cm}^{2} \mathrm{~V}^{-1} \mathrm{~s}^{-1}$ for $\mathrm{Alq}_{2} \mathrm{MHept}$. These experimental values confirm the theoretical predictions $\left(\mu=7.4 \cdot 10^{-8} \mathrm{~cm}^{2} \mathrm{~V}^{-1} \mathrm{~s}^{-1}\right.$ for $\mathrm{Alq}_{3}, \mu=6.2 \times 10^{-6} \mathrm{~cm}^{2} \mathrm{~V}^{-1} \mathrm{~s}^{-1}$ for $\mathrm{Alq}_{2} \mathrm{Acac}$, and $\mu=1.4 \times 10^{-4} \mathrm{~cm}^{2} \mathrm{~V}^{-1} \mathrm{~s}^{-1}$ for $\left.\mathrm{Alq}_{2} \mathrm{MHept}\right)$ and show an improvement of almost four orders of magnitude 

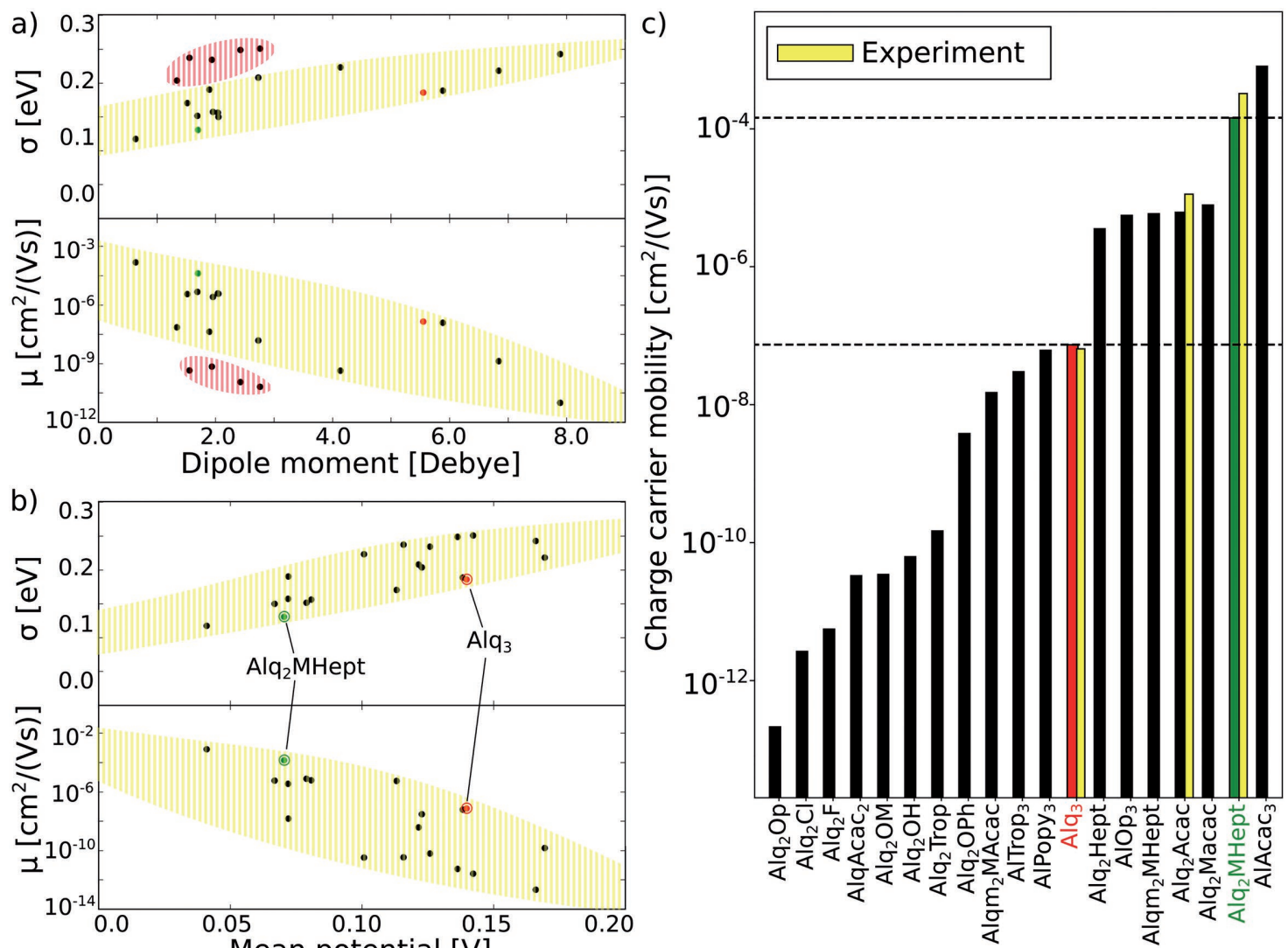

Figure 2. a,b) The energy disorder $\sigma$ and electron mobility $\mu$ of the compounds, compared to their dipole moments and their mean electrostatic potentials. While comparison of dipole moments with mobility and energy disorder (see (a)) leads to a group of outlies marked in red, the mean electrostatic potential (see (b)) correlates better with mobility and energy disorder. c) The calculated electron mobilities of all studied materials. The reference material $\mathrm{Alq}_{3}$ is highlighted in red, $\mathrm{Alq}_{2} \mathrm{MHept}$ as a promising high electron mobility material is marked in green. Its calculated (green bar) and measured (yellow bar) electron mobility is more than 3 orders of magnitude higher than that of Al $q_{3}$. Due to its low electron affinity, AlAcac ${ }_{3}$ is discarded as an ETL candidate. A detailed analysis of the charge carrier mobility is shown in Figure S3 (Supporting Information).

between $\mathrm{Alq}_{3}$ and $\mathrm{Alq}_{2} \mathrm{MHept}$. This result demonstrates that detailed understanding of structure-property relations of materials enables targeted in silico molecule design. To demonstrate the general applicability of the approach to other classes of materials, the same design principles were applied to a stateof-the-art electron conducting material (P4PyMPM) with an electron mobility about three orders of magnitude higher than that of $\mathrm{Alq}_{3}$, leading to a similar boost in electron mobility as Alq2MHept. The theoretical results are shown in Figure S6 (Supporting Information).

In order to substitute $\mathrm{Alq}_{3}$ by a higher mobility material in ETLs, there should be little change in the energy levels to maintain optimal charge injection. The energy level shifts can be directly observed in, e.g., optical measurements or photoelectron spectroscopy. Figure $3 \mathrm{c}-\mathrm{e}$ shows experimental absorption and photoluminescence spectra of $\mathrm{Alq}_{3}$, $\mathrm{Alq}_{2} \mathrm{Acac}$, and $\mathrm{Alq}_{2} \mathrm{M}$ Hept. The absorption maximum of $\mathrm{Alq}_{3}$ is at a wavelength of $\lambda=390 \mathrm{~nm}$ while $\mathrm{Al}_{2}$ Acac and $\mathrm{Al}_{2}$ MHept show maxima at $\lambda=384 \mathrm{~nm}$ and $\lambda=386 \mathrm{~nm}$, respectively (see Figure $3 \mathrm{~d}$ ). This confirms the theoretical findings, where we also observe a slightly blue-shifted IP-EA gap upon substitution of quinoline with smaller $\pi$-conjugated ligands. Similar, but slightly larger, blue-shifts can be observed in the PL peaks shown in Figure 3e, going from $\lambda=523 \mathrm{~nm}$ for $\mathrm{Alq}_{3}$ to $\lambda=511 \mathrm{~nm}$ for $\mathrm{Alq}_{2} \mathrm{Acac}$, and $\lambda=512 \mathrm{~nm}$ for $\mathrm{Alq}_{2} \mathrm{MHept}$.

To confirm the theoretically predicted stability of the IP of $\mathrm{Alq}_{3}, \mathrm{Al}_{2} \mathrm{Acac}$, and $\mathrm{Al}_{2} \mathrm{MHept}$, with calculated IP of 5.34, 5.46, and $5.31 \mathrm{eV}$, respectively, we performed photoelectron yield spectroscopy in air measurements (PESA). We find an ionization potential of IP $=5.85 \mathrm{eV}$ of $\mathrm{Alq}_{3}$ and ionization potentials of IP $=5.88 \mathrm{eV}$ and IP $=5.89 \mathrm{eV}$ for $\mathrm{Alq}_{2} \mathrm{Acac}$ and $\mathrm{Al}_{2} \mathrm{MHept}$, respectively. The IP differences between the materials are within the range of their energy disorder (up to $\sigma=0.19 \mathrm{eV}$ ), while deviations between predicted and measured absolute IPs can be attributed to well-known DFT limitations. ${ }^{[22]}$ Given the stability of the IP and the band gap, we infer stability of the EA.

In conclusion, we demonstrate the predictive power of an in silico multiscale model for key parameters of amorphous organic semiconductors, including charge carrier mobility and energy levels, thereby advancing an emerging trend for virtual materials design. ${ }^{[23]}$ Starting from one of the most widely used ETL materials, our approach allows a systematic improvement of the electron mobility of organic materials, potentially boosting power efficiency by decreasing recombination and driving voltage of contemporary OLEDs. ${ }^{[24]}$ We show that energy disorder and charge mobility strongly depend on the 
a)
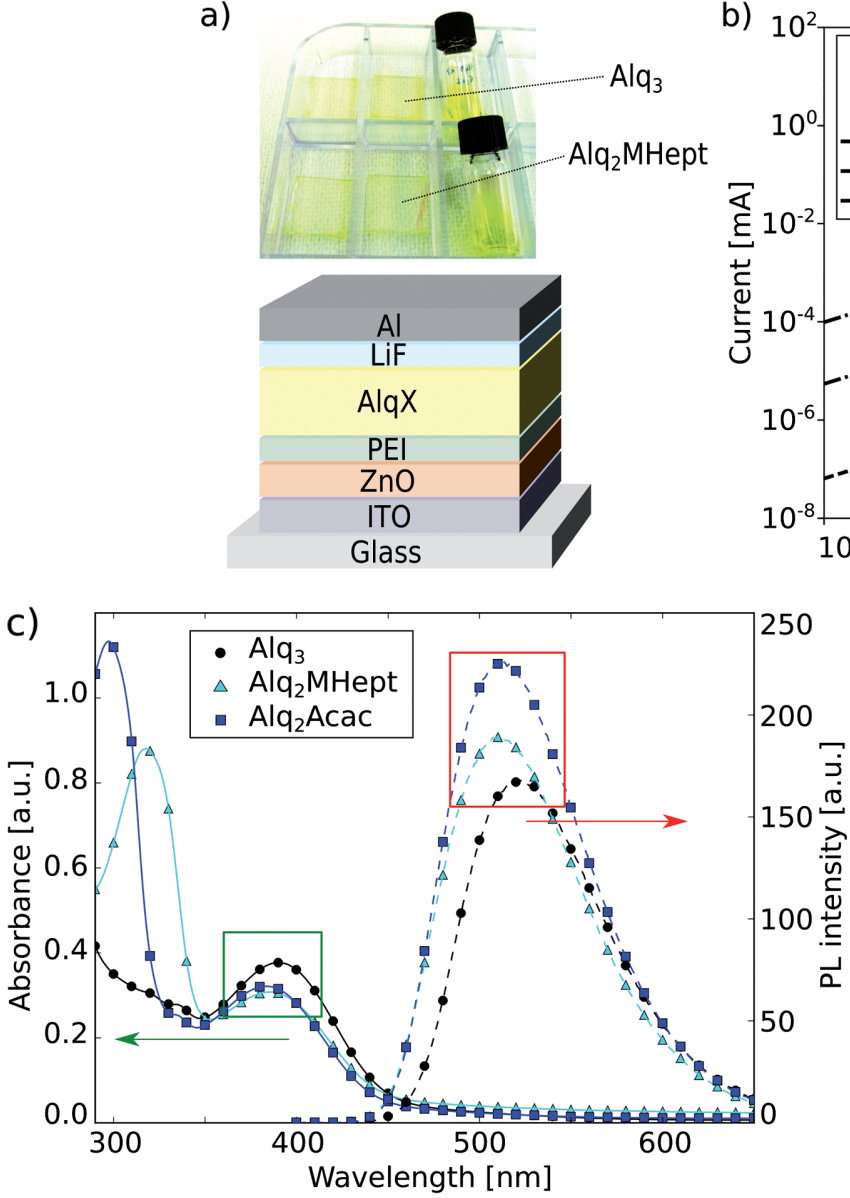

b)

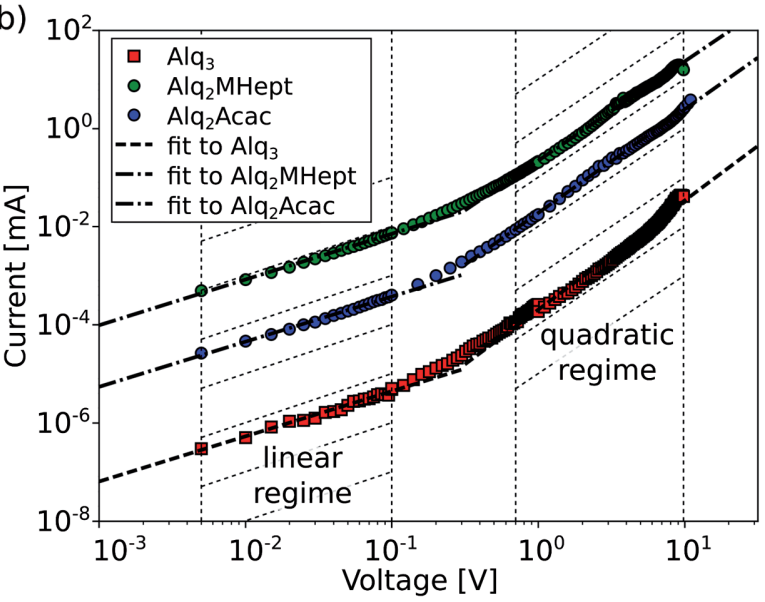

d)

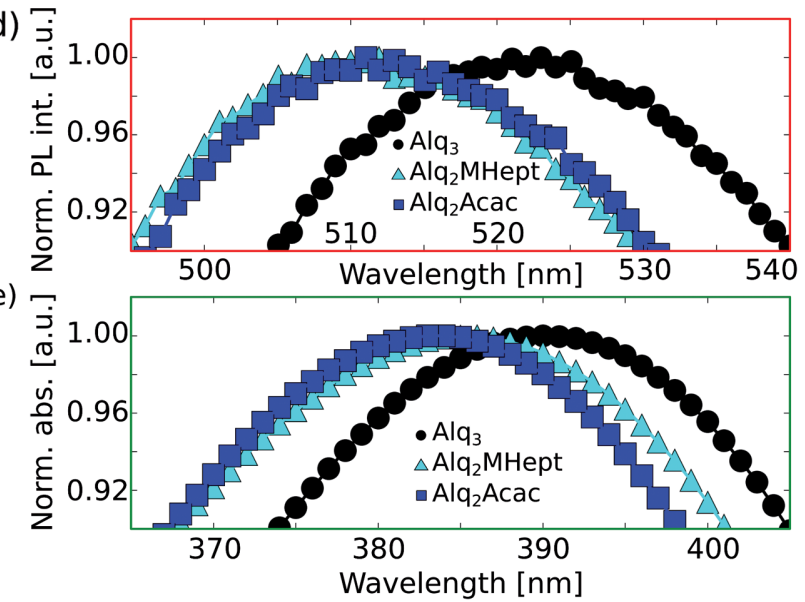

Figure 3. a) Spin-coat layers of $\mathrm{Alq}_{3}$ and $\mathrm{Alq}_{2} \mathrm{MHept}$ as well as the device architecture used for SCLC measurements. b) The current voltage characteristics of $A q_{q_{3}}, A l q_{2} A c a c$, and $A q_{2}$ MHept. A transition from the linear to the quadratic SCLC regime is visible. The vertical dotted lines indicate the range of data points used for extracting the charge carrier mobility. Additional dotted lines with $I \sim U$ and $I \sim U^{2}$ are shown to guide the eye. The dashed/ dashed-dotted lines are fits to the experimental data in the linear/quadratic regime. c) Absorbance (solid lines) and photoluminescence (dashed line) spectra of $\mathrm{Alq}_{3}, \mathrm{Alq}_{2} \mathrm{Acac}$, and $\mathrm{Alq}_{2} \mathrm{MHept}$ are depicted. Absorbance peaks in the $\lambda=380-390 \mathrm{~nm}$ range correspond to the quinolone ligands and the absorbance peak in the $\lambda=300-320 \mathrm{~nm}$ range correspond to the Acac/MHept ligands. Photoluminescence maxima are found in the $\lambda=510-530 \mathrm{~nm}$ range. $d, e)$ The normalized luminescence and absorbance maxima.

near-field electrostatic potential of a molecule, which is not fully captured by the dipole moment. We furthermore demonstrate a computational protocol to control the energy levels of the molecule. Using these design criteria, we develop a new electron conducting material with an electron mobility four orders of magnitude higher than that of the reference material. As our observations apply to amorphous organic semiconductors in general, the design rules established in this work may be helpful to design new materials with improved charge carrier mobility relevant to many applications of organic electronics.

\section{Experimental Section}

Simulations: DFT calculations were carried using the Turbomole Package. ${ }^{[14]}$ All calculations were performed using the hybrid B3-LYP[25] functional. Reorganization energies were calculated using the def2$T Z V P^{[26]}$ basis-set while for energy levels, energy disorder, and electronic couplings, the def2-SV $(\mathrm{P})^{[27]}$ basis-set was used. Atomistically resolved morphologies were generated using the Metropolis Monte Carlo based simulated annealing method DEPOSIT. ${ }^{[13]}$ This method required
DFT-optimized molecular conformations and partial charges (B3-LYP/ def2-SV $(P))$. Energy disorder and HOMO/LUMO levels as well as IPs and EA were calculated using the Quantum Patch method. ${ }^{[16]}$

Characterization: ${ }^{1} \mathrm{H}$ and ${ }^{13} \mathrm{C}$ NMR spectra were recorded using a Bruker FT-NMR Avance III spectrometer with working frequency $500 \mathrm{MHz}$. The residual solvent peak was used as an internal reference. Infrared spectra were recorded in the $4000-400 \mathrm{~cm}^{-1}$ range with a PerkinElmer Spectrum 6× FT-IR spectrophotometer. Elemental analyses of $\mathrm{C}, \mathrm{H}$, and $\mathrm{N}$ were carried out on a Vario Micro Cube. Electrospray ionization (ESI)-time-of-flight (TOF) mass spectrometric analytical data was acquired on a Bruker microOTOF-Q II equipped with a nanospray source. Single crystal $\mathrm{X}$-ray diffraction measurements were made at $180.15 \mathrm{~K}$ on a STOE IPDS2T diffractometer with graphite monochromated Mo $K \alpha$ radiation $(\lambda=0.71073 \AA)$. The structure was solved with the SHELXS program using Patterson Method and refined with the SHELXL program ${ }^{[28]}$ using Least Squares minimization. CCDC1535127 contains the crystallographic data for this paper.

Synthesis: $\mathrm{Al}\left(\mathrm{CH}_{3}\right)_{3}, \mathrm{Al}\left(\mathrm{NO}_{3}\right)_{3}$, 8-hydroxyquinoline, 2,4-pentanedione, piperidine, and 4-methyl-3,5-heptanedione were purchased and used without further purification. Toluene was distilled from sodium beads under argon.

$A l q_{3}$ : It was synthesized following the procedure reported in the literature. [29] 
Alq $q_{2}$ Acac: It was synthesized slightly modifying a procedure reported in the literature: ${ }^{[30]} \mathrm{Al}\left(\mathrm{NO}_{3}\right)_{3} \cdot 9 \mathrm{H}_{2} \mathrm{O}(1.125 \mathrm{~g}, 3 \mathrm{mmol})$ was dissolved in $20 \mathrm{~mL}$ ethanol and heated to $60^{\circ} \mathrm{C}$. A solution of 2,4-pentanedione $(0.31 \mathrm{~mL}, 3 \mathrm{mmol})$ and piperidine $(0.29 \mathrm{~mL}, 3 \mathrm{mmol})$ in $10 \mathrm{~mL}$ ethanol was added dropwise into the above solution and the reaction was stirred for $30 \mathrm{~min}$. Then, a second solution of 8-hydroxyquinoline $(0.870 \mathrm{~g}$, $6 \mathrm{mmol})$ and piperidine $(0.59 \mathrm{~mL}, 6 \mathrm{mmol})$ in $10 \mathrm{~mL}$ ethanol was added dropwise and the mixture was stirred for $4 \mathrm{~h}$. The product was collected by filtration and washed with ethanol and diethyl ether. Yield $1.01 \mathrm{~g}(81 \%)$.

Alq 2 MHept: $\mathrm{Al}\left(\mathrm{CH}_{3}\right)_{3}(2 \mathrm{M}$ toluene, $0.48 \mathrm{~mL}, 1 \mathrm{mmol})$ was added to a $100 \mathrm{~mL}$ three-necked flask with $40 \mathrm{~mL}$ freshly distilled toluene at room temperature under argon. 4-Methyl-3,5-heptanedione $(0.15 \mathrm{~mL}$, $1 \mathrm{mmol}$ ) in $10 \mathrm{~mL}$ freshly distilled toluene was added dropwise. The solution was stirred for $30 \mathrm{~min}$. Then, 8-hydroxyquinoline $(0.290 \mathrm{~g}$, $2 \mathrm{mmol}$ ) in $10 \mathrm{~mL}$ freshly distilled toluene was added dropwise and the mixture was stirred overnight. The solid, which is $\mathrm{Al}_{3}$, was removed by filtration and the solution was concentrated under reduced pressure. Successive recrystallizations of the crude from diethyl ether lead to the desired product. Yield: $0.050 \mathrm{~g}(11 \%)$. ${ }^{1} \mathrm{H}-\mathrm{NMR}\left(500 \mathrm{MHz}, \mathrm{CD}_{2} \mathrm{Cl}_{2}\right.$, $\left.25^{\circ} \mathrm{C}, \delta(\mathrm{ppm})\right): 8.59(\mathrm{~d}, J=4.2 \mathrm{~Hz}, 2 \mathrm{H}), 8.17(\mathrm{~d}, J=8.1 \mathrm{~Hz} 2 \mathrm{H})$, $7.49(\mathrm{t}, J=8.0 \mathrm{~Hz} 2 \mathrm{H}), 7.28$ (dd, $J=4.2$ and $8.1 \mathrm{~Hz}, 2 \mathrm{H}), 7.03(2 \mathrm{xd}$, $J=8.0 \mathrm{~Hz}, 4 \mathrm{H}), 2.34(\mathrm{q}, J=7 . \mathrm{Hz} 4 \mathrm{H}), 1.88(\mathrm{~s}, 3 \mathrm{H}), 0.78(\mathrm{t}, J=7.0 \mathrm{~Hz}$, 6H); ${ }^{13} \mathrm{C}$-NMR $\left(500 \mathrm{MHz}, \mathrm{CD}_{2} \mathrm{Cl}_{2}, 25{ }^{\circ} \mathrm{C}, \delta\right.$ (ppm)): 194.5, 190.2, 159.5, $144.4,139.0,133.2,131.0,129.5,121.9,111.7,111.6,31.4,13.4,9.3$; IR $\left(\mathrm{KBr}\right.$ pellet, $\left.\mathrm{cm}^{-1}\right): 3446 \mathrm{~m}, 1589 \mathrm{~s}, 1579 \mathrm{~s}, 1499 \mathrm{vs}, 1472 \mathrm{vs}, 1383 \mathrm{~s}, 1286 \mathrm{~s}$, $1116 \mathrm{~s}, 1029 \mathrm{~m}, 826 \mathrm{~s}, 752 \mathrm{~s}, 650 \mathrm{~m}, 547 \mathrm{~m}$; elemental analysis (calcd, found for $\left.\mathrm{C}_{26} \mathrm{H}_{25} \mathrm{AlN}_{2} \mathrm{O}_{4}, \mathrm{MW}=456.48 \mathrm{~g} \mathrm{~mol}^{-1}\right)$ : C $(68.41,68.2), \mathrm{H}(5.52,5.5)$, $\mathrm{N}(6.14,6.0)$; ESI-mass spectrocopy (MS) $\left(\mathrm{CH}_{2} \mathrm{Cl} 2, \mathrm{~m} / \mathrm{z}\right.$, rel. intensity, assigned structure): $457.1617\left(100 \%,[\mathrm{M}+\mathrm{H}]^{+}\right.$, calcd. $\left.=457.1639\right)$, 312.1329 (68.30\%, Alq(MHept) ${ }^{+}$, calcd. $\left.=312.1175\right)$.

Mobility Measurements: For SCLC measurements, electrononly devices were built according to the architecture depicted in Figure 3a (glass substrate/indium tin oxide (ITO) $(125 \mathrm{~nm}) / \mathrm{ZnO}+$ polyethyleneimine, PEI) $(15 \mathrm{~nm}) / \mathrm{AlqX} / \operatorname{LiF}(1 \mathrm{~nm}) / \mathrm{Al}(200 \mathrm{~nm})$, with $\mathrm{AlqX}=\mathrm{Alq}_{3}$ or $\mathrm{Alq}_{2} \mathrm{MHept}$. ITO coated glass substrates $\left(R_{\mathrm{sq}} \approx 15 \Omega \mathrm{sq}^{-1}\right)$ were structured in hydrochloric acid and sequentially cleaned in acetone and isopropanol in an ultrasonic bath $(10 \mathrm{~min})$. $\mathrm{ZnO}$ nanoparticles (Nanograde $\mathrm{N}-10)$ were spin cast (4000 rpm, $30 \mathrm{~s})$ from isopropanol dispersion $(0.55 \mathrm{wt} \%)$ in nitrogen atmosphere, followed by thermal annealing $\left(80^{\circ} \mathrm{C}, 10 \mathrm{~min}\right)$, resulting in a thickness of $15 \mathrm{~nm}$. For an efficient electron injection, a layer of PEI was spin coated atop $(4000 \mathrm{rpm}, 30 \mathrm{~s})$ from 2-methoxyethanol solution $\left(3.9 \mathrm{~g} \mathrm{~L}^{-1}\right)$ under nitrogen atmosphere. The substrates were then annealed in ambient atmosphere $\left(100{ }^{\circ} \mathrm{C}, 10 \mathrm{~min}\right)$ and rinsed with ethanol $(30 \mathrm{~s})$ to remove excess PEI. The aluminum-quinoline layer was prepared by dissolving $\mathrm{Al}_{3}$ in chloroform $\left(20 \mathrm{~g} \mathrm{~L}^{-1}\right)$, Al $\mathrm{q}_{2} \mathrm{Acac}$ in dichloromethane $\left(20 \mathrm{~g} \mathrm{~L}^{-1}\right)$, and $\mathrm{Alq}_{2} \mathrm{MHept}$ in dichloromethane $\left(20 \mathrm{~g} \mathrm{~L}^{-1}\right)$, respectively, and spin casting $(800 \mathrm{rpm}, 40 \mathrm{~s})$ in ambient atmosphere. The substrates were then transferred to a vacuum chamber $\left(10^{-6} \mathrm{mbar}\right)$ to deposit the counter electrode of LiF $(1 \mathrm{~nm})$ and $\mathrm{Al}(200 \mathrm{~nm})$ by thermal evaporation. Current density-voltage $(U-V)$ characteristics were recorded using a source measure unit (Keithley 238) at room temperature under nitrogen atmosphere. The thickness of the layers was determined using a tactile stylus profiler (Dektak XT, Bruker).

Absorbance, Photoluminescence and Photoelectron Yield: For measurements of absorbance, photoluminescence, and photoelectron yield, aluminum-quinoline thin films were spin cast on glass substrates according to the procedure described above. Absorbance spectra were measured on an UV-vis-NIR spectrophotometer (Cary5000, Agilent Technologies). Photoluminescence spectra were recorded on a fluorescence spectrophotometer (Cary Eclipse, Agilent Technologies), with a chosen excitation wavelength of $385 \mathrm{~nm}$. The IPs were determined by photo-electron yield spectroscopy in air (PESA) (AC-2E, Rieken Keiki).

Atomic Force Microscopy (AFM): AFM (Dimension ICON, Bruker) images were recorded on thin films on glass substrates in tapping mode.

CCDC-1535127 contains the supplementary crystallographic data for this paper. These data can be obtained free of charge from The
Cambridge Crystallographic Data Centre via www.ccdc.cam.ac.uk/ data_request/cif.

\section{Supporting Information}

Supporting Information is available from the Wiley Online Library or from the author.

\section{Acknowledgements}

The authors acknowledge funding by the Helmholtz program "Science and Technology of Nanosystems" (STN), the EXTMOS EU project (grant number: 646176), the Transregio "3Met" Project C5 and the CarlZeiss Foundation for funding the project "Multiskalen Modellierung elektronischer Eigenschaften von Materialien in der organischen Elektronik". The calculations were performed on the computational resources bwUniCluster and ForHLR Phase I funded by the Ministry of Science, Research and the Arts Baden-Württemberg and DFG ("Deutsche Forschungsgemeinschaft"). UV-vis-NIR absorption spectrophotometry and PESA were made available by the Federal Ministry of Education and Research (BMBF) under contract 03EK3504 (project TAURUS).

\section{Conflict of Interest}

The authors declare no conflict of interest.

\section{Keywords}

charge mobility, computational material design, multiscale modeling, organic electronics, organic semiconductors

[1] a) T.-H. Lai, J. W. Lee, J. R. Manders, F. So, Sci. Rep. 2014, 4, 5946; b) J. Zou, K. Zhang, J. Li, Y. Zhao, Y. Wang, S. K. R. Pillai, H. V. Demir, X. Sun, M. B. Chan-Park, Q. Zhang, Sci. Rep. 2015, 5, 11755.

[2] C. W. Tang, S. VanSlyke, Appl. Phys. Lett. 1987, 51, 913.

[3] a) S. Naka, H. Okada, H. Onnagawa, Y. Yamaguchi, T. Tsutsui, Synth. Met. 2000, 111, 331; b) S. Tse, K. Kwok, S. So, Appl. Phys. Lett. 2006, 89, 262102.

[4] a) S. Naka, H. Okada, H. Onnagawa, T. Tsutsui, Appl. Phys. Lett. 2000, 76, 197; b) P. Kathirgamanathan, S. Surendrakumar, R. R. Vanga, S. Ravichandran, J. Antipan-Lara, S. Ganeshamurugan, M. Kumaraverl, G. Paramaswara, V. Arkley, Org. Electron. 2011, 12, 666.

[5] D. Yokoyama, H. Sasabe, Y. Furukawa, C. Adachi, J. Kido, Adv. Funct. Mater. 2011, 21, 1375.

[6] M. Pfeiffer, K. Leo, X. Zhou, J. Huang, M. Hofmann, A. Werner, J. Blochwitz-Nimoth, Org. Electron. 2003, 4, 89.

[7] H. Bässler, Phys. Status Solidi B 1981, 107, 9.

[8] A. Dieckmann, H. Bässler, P. Borsenberger, J. Chem. Phys. 1993, 99, 8136.

[9] a) W. F. Pasveer, J. Cottaar, C. Tanase, R. Coehoorn, P. A. Bobbert, P. W. M. Blom, D. M. De Leeuw, M. A. J. Michels, Phys. Rev. Lett. 2005, 94, 206601; b) J. Cottaar, L. Koster, R. Coehoorn, P. Bobbert, Phys. Rev. Lett. 2011, 107, 136601; c) R. Coehoorn, W. Pasveer, 
P. Bobbert, M. Michels, Phys. Rev. B 2005, 72, 155206; d) A. Massé, R. Coehoorn, P. Bobbert, Phys. Rev. Lett. 2014, 113, 116604; e) I. Fishchuk, A. Kadashchuk, S. Hoffmann, S. Athanasopoulos, J. Genoe, H. Bässler, A. Köhler, Phys. Rev. B 2013, 88, 125202.

[10] H. Bässler, Phys. Status Solidi B 1993, 175, 15.

[11] a) J. J. Kwiatkowski, J. Nelson, H. Li, J. L. Bredas, W. Wenzel, C. Lennartz, Phys. Chem. Chem. Phys. 2008, 10, 1852; b) P. Kordt, J. J. van der Holst, M. Al Helwi, W. Kowalsky, F. May, A. Badinski, C. Lennartz, D. Andrienko, Adv. Funct. Mater. 2015, 25, 1955.

[12] P. Friederich, V. Meded, A. Poschlad, T. Neumann, V. Rodin, V. Stehr, F. Symalla, D. Danilov, G. Lüdemann, R. Fink, I. Kondov, F. von Wrochem, W. Wenzel, Adv. Funct. Mater. 2016, 26, 5757.

[13] T. Neumann, D. Danilov, C. Lennartz, W. Wenzel, J. Comput. Chem. 2013, 34, 2716.

[14] R. Ahlrichs, M. Bär, M. Häser, H. Horn, C. Kölmel, Chem. Phys. Lett. 1989, 162, 165.

[15] J. J. P. Stewart, J. Comput.-Aided Mol. Des. 1990, 4, 1.

[16] P. Friederich, F. Symalla, V. Meded, T. Neumann, W. Wenzel, J. Chem. Theory Comput. 2014, 10, 3720.

[17] P. Friederich, V. Meded, F. Symalla, M. Elstner, W. Wenzel, J. Chem Theory Comput. 2015, 11, 560.

[18] V. Stehr, J. Pfister, R. F. Fink, B. Engels, C. Deibel, Phys. Rev. B 2011, 83, 155208.

[19] S. F. Nelsen, S. C. Blackstock, Y. Kim, J. Am. Chem. Soc. 1987, 109, 677.
[20] V. Rodin, F. Symalla, P. Friederich, V. Meded, D. Danilov, A. Poschlad, G. Nelles, F. von Wrochem, W. Wenzel, Phys. Rev. B 2015, 91, 155203.

[21] R. L. Martin, J. D. Kress, I. H. Campbell, D. L. Smith, Phys. Rev. B 2000, 61, 15804

[22] X. Blase, C. Attaccalite, V. Olevano, Phys. Rev. B 2011, 83, 115103.

[23] a) M. Schwarze, W. Tress, B. Beyer, F. Gao, R. Scholz, C. Poelking, K. Ortstein, A. A. Günther, D. Kasemann, D. Andrienko, Science 2016, 352, 1446; b) R. Gómez-Bombarelli, J. Aguilera-Iparraguirre, T. D. Hirzel, D. Duvenaud, D. Maclaurin, M. A. Blood-Forsythe, H. S. Chae, M. Einzinger, D.-G. Ha, T. Wu, Nat. Mater. 2016, 15, 1120.

[24] H. Sasabe, E. Gonmori, T. Chiba, Y.-J. Li, D. Tanaka, S.-J. Su, T. Takeda, Y.-J. Pu, K.-i. Nakayama, J. Kido, Chem. Mater. 2008, 20, 5951.

[25] A. D. Becke, J. Chem. Phys. 1993, 98, 1372.

[26] A. Schafer, C. Huber, R. Ahlrichs, J. Chem. Phys. 1994, 100, 5829.

[27] A. Schafer, H. Horn, R. Ahlrichs, J. Chem. Phys. 1992, 97, 2571.

[28] G. M. Sheldrick, Acta Crystallogr., Sect. C: Struct. Chem. 2015 71,3 .

[29] H. Li, F. Zhang, Y. Wang, D. Zheng, J. Mater. Sci. Eng. B 2003, 100, 40.

[30] B. Xu, L. Chen, X. Liu, H. Zhou, H. Xu, X. Fang, Y. Wang, Appl. Phys. Lett. 2008, 92, 103305. 


\section{Repository KITopen}

Dies ist ein Postprint/begutachtetes Manuskript.

Empfohlene Zitierung:

Friederich, P.; Gómez, V.; Sprau, C.; Meded, V.; Strunk, T.; Jenne, M.; Magri, A.; Symalla, F.; Colsmann, A.; Ruben, M.; Wenzel, W.

Rational In Silico Design of an Organic Semiconductor with Improved Electron Mobility. 2017. Advanced materials doi: $10.5445 / I R / 1000076156$

Zitierung der Originalveröffentlichung:

Friederich, P.; Gómez, V.; Sprau, C.; Meded, V.; Strunk, T.; Jenne, M.; Magri, A.; Symalla, F.; Colsmann, A.; Ruben, M.; Wenzel, W.

Rational In Silico Design of an Organic Semiconductor with Improved Electron Mobility. 2017. Advanced materials, Art.Nr.: 1703505.

doi:10.1002/adma.201703505 